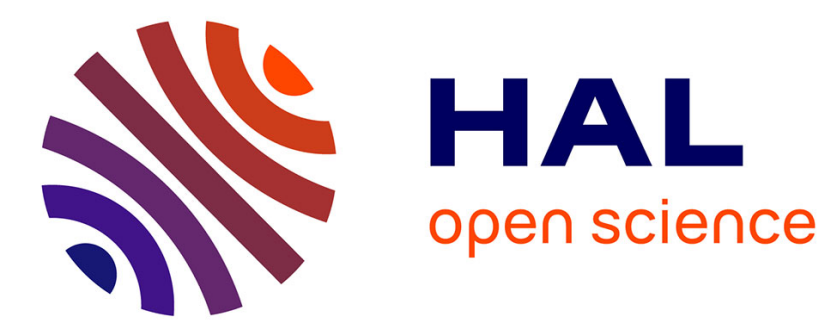

\title{
Les causes démographiques de la crise du logement
}

Gérard-François Dumont

\section{To cite this version:}

Gérard-François Dumont. Les causes démographiques de la crise du logement. Informations sociales, 2014, 183, pp.26-34. 10.3917/inso.183.0026 . halshs-01059399v2

\section{HAL Id: halshs-01059399 \\ https://shs.hal.science/halshs-01059399v2}

Submitted on 15 Sep 2014

HAL is a multi-disciplinary open access archive for the deposit and dissemination of scientific research documents, whether they are published or not. The documents may come from teaching and research institutions in France or abroad, or from public or private research centers.
L'archive ouverte pluridisciplinaire HAL, est destinée au dépôt et à la diffusion de documents scientifiques de niveau recherche, publiés ou non, émanant des établissements d'enseignement et de recherche français ou étrangers, des laboratoires publics ou privés. 


\section{Les causes démographiques de la crise du logement}

Gérard-François Dumont - géographe, économiste et démographe

La fin de la crise du logement en France n'est pas pour demain. La demande de logements va s'intensifier en raison de l'accroissement prévisible de la population mais aussi d'autres facteurs que la démographie permet de comprendre. Cette augmentation numérique se répartit très inégalement dans les territoires tandis que la croissance du nombre de ménages est plus rapide que celle la population - et est appelée à le demeurer au vu de l'évolution de la composition de ceux-ci.

Selon la tendance actuelle, le parc de logements français demeure inadapté aux besoins ou insuffisant. Au-delà des critiques susceptibles d'être portées sur le rôle des politiques publiques françaises du logement, l'analyse démographique livre des bases objectives permettant de comprendre le besoin de logements. En effet, ce dernier découle tout d'abord de l'augmentation de la population de la France, donc du nombre de personnes à loger. Mais il tient aussi au fait que cette augmentation, loin de s'effectuer de façon homothétique sur le territoire, connaît d'importantes variations, notamment sous l'effet des choix géographiques des migrations internes et internationales. Une autre explication démographique du besoin de logements est l'évolution du nombre de ménages, qui n'est pas non plus homothétique à la population, notamment en raison du vieillissement de la population. Par ailleurs se constate une inadéquation géographique entre les territoires où l'on dispose de logements et ceux où les besoins sont les plus intenses.

Une population croissante à loger

Le nombre d'habitants que la France métropolitaine doit loger augmente de façon continue au fil des années, à l'inverse d'une dizaine de pays européens dont la population diminue, comme la Bulgarie, l'Espagne, le Portugal ou la Roumanie. Cette augmentation s'explique par les deux composantes de 
l'accroissement démographique : le solde naturel et le solde migratoire (voir graphique 1). De 2000 à 2012, la population de la France s'est accrue par un solde naturel de 3,2 millions d'habitants, sous deux effets. D'une part, la natalité de la France est stimulée par une fécondité qui est nettement supérieure à la moyenne européenne et qui s'applique sur des générations en âge de féconder plus nombreuses.

D'autre part, la mortalité, en dépit du vieillissement de la population, se trouve notamment limitée par les progrès dans l'espérance de vie des personnes âgées. Quant au solde migratoire évalué par l'Insee (1), plus élevé depuis les années 2000 que dans les années 1990, son montant cumulé des années 2000 à 2012 dépasse légèrement un million.

\section{Graphique 1. Le solde naturel, le solde migratoire et l'accroissement total de la population en France}

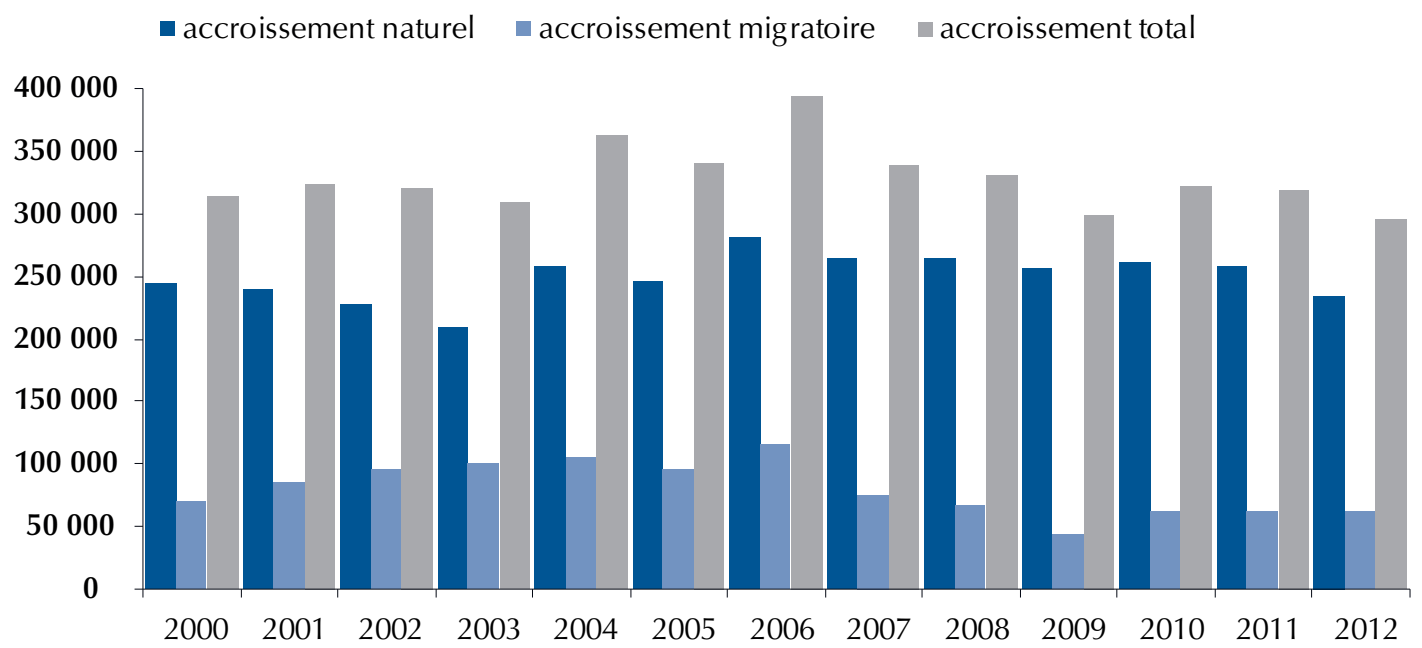

Source : chiffres Insee métropole.

La connaissance de ces données permet de proposer des projections démographiques bénéficiant de la logique d'inertie des phénomènes de population. Les projections moyennes, fondées sur une légère réduction de la différence d'espérance de vie entre hommes et femmes, sur la fécondité actuelle et sur le maintien d'un courant d'immigration équivalent à celui des années 2000, laissent penser que la population de la France métropolitaine pourrait augmenter de façon continue dans le futur jusqu'à 70 millions d'habitants à l'horizon 2040 (2), soit un besoin à venir de logements pour plusieurs millions d'habitants supplémentaires. Mais les besoins de logements engendrés par cette croissance démographique constatée et projetée sont inégaux selon les territoires. En effet, les migrations internationales sont géographiquement très concentrées et les migrations résidentielles internes privilégient certains territoires au détriment d'autres. 


\section{Migrations et inégalités géographiques des besoins en logement}

S’il est vrai que le solde naturel est différencié selon les territoires, et engendre donc des besoins variés de logement, le facteur majeur qui influe sur ces besoins est le mouvement migratoire dans ses composantes internationale et interne. La géographie de l'immigration en France présente une très forte concentration avec, selon les estimations, les deux cinquièmes des migrants qui s'installent dans la seule région Île-de-France, quel que soit leur continent d'origine.

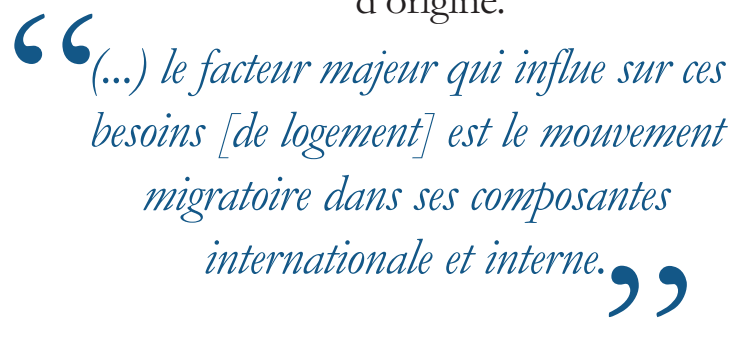

En conséquence, une demande plus forte de logement liée à la migration internationale vient s'exercer sur les territoires franciliens considérés comme les plus attractifs par les immigrants internationaux.

L'effet des migrations internes se combine aux migrations internationales pour accentuer la pression sur certains marchés locaux du logement et en délaisser d'autres. Mais le jeu des migrations résidentielles internes est fort différent de celui des migrations internationales. D'une part, il fait de l'Île-de-France un territoire répulsif, dont le solde migratoire est négatif et dont le besoin en logements s'accroît donc uniquement par son solde naturel fortement positif. D'autre part, il est très corrélé avec l'âge, ce qu'il importe de préciser. Ainsi, de 18 à 30 ans (3), les jeunes vivent une double instabilité de statut social. Ils prolongent la durée de leurs études puis enchaînent souvent une série d'emplois divers avant de pouvoir accéder à un $\mathrm{CDI}{ }^{(4)}$. En même temps, les couples se forment et se séparent très librement. Cette tranche d'âge se concentre fortement dans les villes universitaires et recherche des petits logements locatifs.

Autour de la trentaine, les jeunes couples se stabilisent doublement. L'accès à deux CDI, condition pour accéder à la propriété, ouvre la possibilité de réaliser le projet d'avoir des enfants. Ces couples plébiscitent le marché des logements périurbains, sur des territoires bâtis en continuité des grandes villes, ou celui des pavillons para-urbains, sur des territoires situés en périphérie des agglomérations et de morphologie essentiellement rurale, où ils représentent $90 \%$ des acquéreurs. Ce séjour dans le périurbain ou le paraurbain dure jusqu'à la fin des études des enfants, donc vers la soixantaine et la proximité de la retraite. Dans la catégorie des couples actifs, on compte aussi environ plusieurs centaines de milliers de couples biactifs qui disposent de deux résidences permanentes, parce que leurs lieux de travail respectifs sont situés dans des villes trop éloignées l'une de l'autre.

Avec la retraite et jusque vers 80 ans, deux tendances principales s'observent. Des jeunes retraités, en bonne santé et dotés de revenus réguliers, reviennent en ville pour profiter des loisirs urbains, se rapprocher de leurs enfants ou œuvrer dans des associations. Un autre flux (5) se dirige vers le 
logement de la période de retraite ; son emplacement est celui d'une résidence secondaire antérieure ou est choisi notamment en fonction des préférences de loisirs, pour accueillir la famille pendant les week-ends et les vacances, ou de l'origine géographique. Prenons l'exemple de la Bretagne pour illustrer ces différents choix. Parmi les personnes s'y rendant à l'âge de la retraite, moins d'une sur deux est née dans la région; ce n'est donc pas que du retour, l'installation étant souvent liée au désir de venir passer sa retraite dans le lieu où l'on venait passer ses vacances.

Dans les dernières années de la vie peut venir l'âge d'une dépendance croissante et le temps d'arbitrages difficiles entre maintien à domicile, solution la plus souhaitée, l'accueil au logement d'un tiers et les résidences spécialisées. Ainsi, les migrations contribuent à différencier considérablement, selon les territoires, le besoin de logement. En fait, ce besoin n'est pas celui des habitants mais de ce qu'on appelle un ménage ${ }^{(6)}$, c'est-à-dire par une personne ou un ensemble de personnes habitant une résidence principale, qu'elles aient ou non des liens de parenté. Le besoin de logement principal est donc exactement corrélé avec celui des ménages, puisqu'il y a un logement par ménage. La connaissance de l'évolution du nombre de ménages est donc essentielle.

\section{Une hausse de ménages \\ supérieure à celle de la population}

Les recensements montrent que le nombre de ménages, donc le besoin de résidences principales, augmente beaucoup plus vite que la population : entre 1999 et 2010 (7), la population de la France métropolitaine a augmenté de $7,1 \%$ et le nombre de ménages de 13,9\%. Il a donc fallu que le nombre de résidences principales s'accroisse de ce denier pourcentage. Cet écart entre le taux de croissance de la population et celui des ménages s'explique par la baisse du nombre de personnes par ménage. Ce dernier est passé de 2,4 personnes en 1999 à 2,26 en $2010^{(8)}$, une évolution à la baisse qui prolonge celle des périodes intercensitaires précédentes puisque le nombre de personnes par ménage était de 2,88 en 1975 (voir le graphique 2).

Une telle diminution du nombre de personnes par ménage résulte, tout d'abord, de l'augmentation des ménages composés d'une personne - plus de 1,8 million entre 1999 et 2010 (voir le graphique 3), ce qui traduit l'« effritement » des modes traditionnels de cohabitation, c'est-à-dire, pour aller vite, une désaffection, voulue ou subie, pour la vie en couple. La hausse des ménages d'une seule personne tient à trois facteurs. Tout d'abord, il y a des personnes qui ne se mettent pas en couple. C'est souvent le cas de jeunes adultes. En effet, ces jeunes quittent le domicile parental de moins en moins fréquemment pour se mettre en couple et de plus en plus pour vivre d'abord une période en solitaire, au moins transitoire, ce qui influe naturellement sur la taille des ménages. En outre - et cela est important en matière de logement 


\section{Graphique 2. Évolution du nombre de ménages et de personnes par ménage}

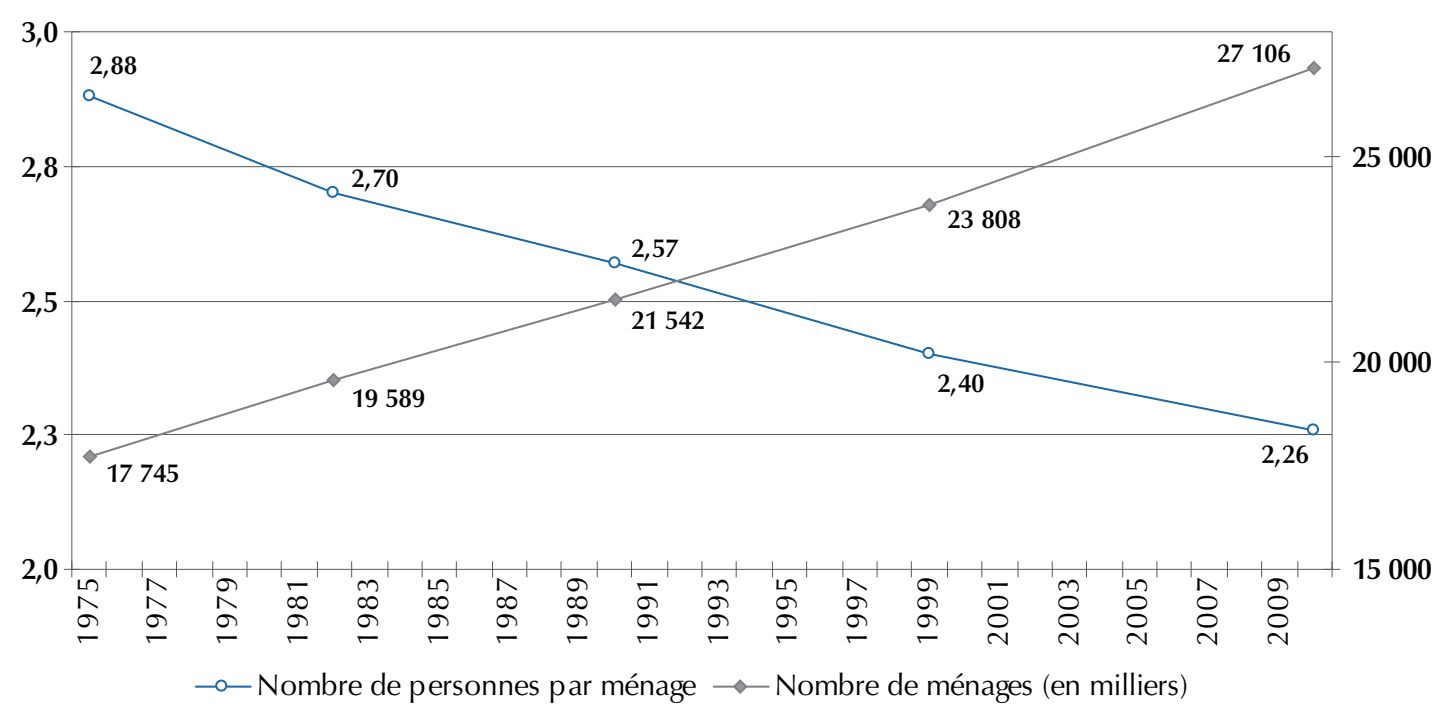

Source : chiffres Insee (France métropole).

- la prise d'un premier logement n'est pas financièrement autonome mais de plus en plus fréquemment aidée par les parents. Cela est lié à la progression du nombre des étudiants dans les années 1990, comme aux aides personnelles au logement qui ont permis aux étudiants ou à leurs familles de bénéficier d'une aide financière dans un contexte où les règles fiscales facilitaient la construction de résidences d'étudiants. En deuxième lieu, l'augmentation des ménages d'une personne est la conséquence de ruptures dans la vie de couple. Chez les couples non mariés, le divorce et les séparations progressent. L'indicateur de divortialité, qui était de 33\% au début des

$\boldsymbol{6} \boldsymbol{G}_{\text {(...) la diminution du nombre de }}$ personnes par ménage [s'explique par] la hausse du nombre des couples sans enfants, (...) principalement (...) celle des ménages de personnes âgées (...). années 1990, a presque toujours dépassé $45 \%$ depuis 2005 : ce n'est plus désormais un mariage sur trois qui aboutit à un divorce, mais près d'un sur deux. Enfin, le veuvage est la troisième cause de la hausse du nombre des ménages d'une personne.

La deuxième composante engendrant la diminution du nombre de personnes par ménage est la hausse du nombre des couples sans enfants, qui s'explique principalement par celle des ménages de personnes âgées, sous le double effet de l'héritage démographique, donc un effet de structure engendrant à lui seul une gérontocroissance, et de l'augmentation de l'espérance de vie des personnes âgées rappelée ci-dessus.

En troisième lieu, un autre marqueur significatif de la diminution du nombre de couples en ménage est la hausse des foyers monoparentaux, dans la mesure où, parmi eux, ceux composés d'un parent et d'un seul enfant entraînent à la baisse le nombre de personnes par ménage. 


\section{Graphique 3. La variation de la composition des ménages en France}
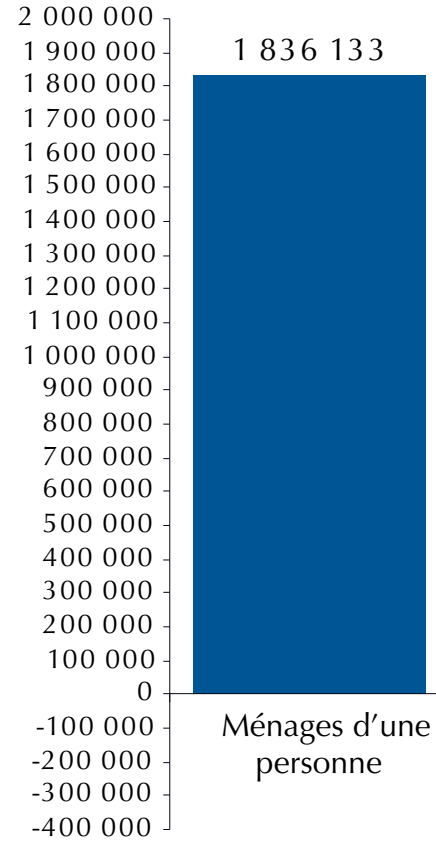

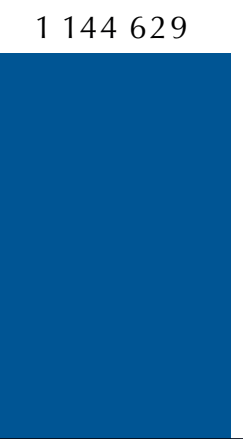

Un couple sans enfant

Source : chiffres Insee RP 2010, RGP 1999 métropole.

Un quatrième facteur est la hausse des « autres ménages sans famille», expression qui recouvre, selon la définition de l'Insee, des «ménages composés de plusieurs personnes isolées ", comme les étudiants en colocation. Enfin, la baisse du nombre de personnes par ménage tient à une dernière raison, la diminution du nombre de ménages composés d'un couple avec un ou plusieurs enfants, soit 340000 de moins entre 1999 et 2010.

Pour le futur, la diminution du nombre de personnes par ménage est la perspective courante (9) pour deux raisons, l'une structurelle et l'autre liée aux comportements se traduisant par la préférence, voulue ou subie, pour la vie en solitaire.

La raison structurelle vient d'un effet mécanique de la pyramide des âges qui accroît le nombre de ménages de personnes âgées, seules ou en couple. Concernant les comportements de cohabitation des individus, il est évidemment délicat de projeter leur évolution. Il y a probablement encore une marge de progression pour la vie en solo, pour des dissolutions de couples plus fréquentes et pour le choix de ne pas vivre en couple en général. Il semble en effet exister, selon la terminologie économique, des « coûts de promiscuité », des « coûts » spécifiquement générés par la vie à deux, c'est-à-dire non des coûts monétaires, mais les effets externes négatifs ressentis du fait des obligations que signifient pour l'individu le fait de vivre avec une autre personnes, même si la cohabitation présente aussi des avantages économiques, notamment des économies d'échelle. Bien que les économies d'échelle liées à la mise en couple ou à la présence d'enfants semblent plus importantes 
aujourd'hui que par le passé, les gens semblent disposés à payer pour ne pas avoir à subir ces « coûts de promiscuité ». C'est, d'un point de vue du besoin en nombre de logements, peut-être inquiétant mais c'est une réalité, à moins que la crise économique, restreignant le pouvoir d'achat, contraigne à des cohabitations « de raison ».

Sous cette dernière réserve, le nombre de personnes par ménage, de 2,26 au recensement de 2010, pourrait passer à 2,1 en 2030, soit une baisse qui s'inscrit dans une tendance séculaire. Dans cette hypothèse, le nombre de ménages passerait de 27,1 à près de 30 millions en 2030, nécessitant en conséquence une augmentation de 10,7 \% du nombre de logements. Les autres hypothèses possibles, y compris celle d'une diminution moins rapide du nombre de personnes par ménage, projettent également une hausse du besoin de logements parce que l'essentiel de la baisse projetée du nombre de personnes par ménage est due aux déformations de la pyramide des âges et non à l'évolution des modes de comportement de cohabitation à sexe et âge donnés.

La question des besoins en logement revêt un autre aspect géodémographique. En effet, en poussant à l'extrême le raisonnement, si tous les logements futurs construits étaient des résidences secondaires, la question des besoins en résidences principales demeurerait entière.

\section{Inadéquation entre géographie des logements et géographie de la population}

En réalité, la France compte davantage de logements que de ménages : 9,6\% des logements, soit 3,1 millions, sont des résidences secondaires ou des logements occasionnels. Ce pourcentage, au recensement de 2010, est supérieur de presque 3 points à celui du recensement de 1968, mais du même ordre de grandeur qu'à celui de 1999, ce qui signifie 300000 résidences secondaires de plus entre 1999 et 2010 soit, ceteris paribus, autant de logements supplémentaires, mais non disponibles comme résidences principales. En outre, même si ces résidences secondaires connaissaient toutes une mutation les conduisant à répondre à la demande de résidences principales, la question du logement ne serait pas automatiquement réglée.

Par ailleurs, environ $7 \%$ des logements étaient vacants en 2010, pourcentage normal compte tenu des multiples causes d'inoccupation d'un logement constatée lors des opérations de recensement (logement proposé à la vente ou à la location ; déjà attribué à un acheteur ou un locataire et en attente d'occupation en raison par exemple de travaux de réhabilitation; en attente de règlement de succession; conservé par un employeur pour un usage futur au profit d'un de ses employés; non habité parce que l'occupant est en mission à l'étranger ou en établissement de soins ; gardé vacant par le propriétaire et sans affectation précise comme un logement très vétuste...). En effet, la tendance est à la spécialisation des territoires : les territoires qui 
comportaient relativement peu de résidences secondaires voient leur proportion diminuer tandis que ceux qui en comportaient une part importante voient leur proportion augmenter. Les territoires où les résidences secondaires ont un fort impact sur le logement sont ceux de toute la façade atlantique, le Sud-Ouest intérieur, avec une sorte de crête allant de la Charente au Gard en passant le sud et l'ouest du Massif central, le massif alpin, le massif pyrénéen ainsi que, plus globalement, le Massif central et le Jura, mais de manière moins marquée.

Ailleurs, notamment dans des rayons très larges autour des grandes villes, par exemple à $50 \mathrm{~km}$ autour de Lyon, on trouve de moins en moins de résidences secondaires, certaines s'étant converties en résidences principales avec le développement de la mobilité. Le massif alpin accroit incontestablement la pression à la demande, d'autant plus que le foncier y est rare. Comme les résidences secondaires ne sont pas localisées là où le besoin de résidences principales est le plus intense, il n'y a pas d'amélioration significative de l'offre possible par cette voie.

En conclusion, la démographie, et plus précisément la géographie de la population, livre donc deux enseignements, l'un méthodologique et l'autre factuel.

Au plan méthodologique, elle permet, à travers ses différents indicateurs dont certains ont été présentés ci-dessus, de nourrir un tableau de bord très utile pour déceler les besoins en logement et leur évolution.

Au plan des faits, l'analyse des projections livre une quasi-certitude : le besoin de logements va augmenter en France dans les années futures en raison de la hausse projetée du nombre de ménages, dont la majeure partie est de nature structurelle, donc indépendante de l'évolution des comportements de cohabitation. En même temps, le besoin en logements doit se traduire par une offre quantitative très différenciée selon les territoires, ce qui signifie que l'enjeu est fort en matière d'aménagement du territoire jusqu'aux niveaux géographiques les plus fins.

\section{Notes}

1 - Voir notamment le périodique annuel Insee Résultats sur la situation démographique.

2 - Insee Première, $\mathrm{n}^{\circ} 1326$.

3 - Ces chiffres ne sont que les moyennes dans une distribution assez dispersée.

4 - Contrat de travail à durée indéterminée. 


\section{Démographie et protection sociale}

Besoins sociaux et démographie

5 - Qui participe notamment du renouveau rural. Voir Pierre Pistre, "Les campagnes françaises : un renouveau incontestable, mais très inégal », Population \& Avenir, n 715, novembre-décembre 2013.

6 - Ne font pas partie des ménages les personnes vivant dans des habitations mobiles (y compris les mariniers), les personnes sans abri et la population des communautés (internat, maisons de retraite, prisons... )

7 - Rappelons que la nouvelle méthode de recensement mise en place en France en 2004 a remplacé le recensement général de la population (RGP) par un recensement de la population (RP).

8 - Il s'agit d'une moyenne car le jeu des types migratoires selon les territoires fait que ce nombre, qui décroît en moyenne, peut croître ici ou là. Voir Jean-François Léger, "Logement et territoire : I'effet très inégal des constructions sur la croissance démographique », Population \& Avenir, $\mathrm{n}^{\circ} 711$, janvier-février 2013.

9 - Gérard-François Dumont (direction), Populations et territoires de France en 2030, le scénario d'un futur choisi, Paris, L'Harmattan, 2008. 